\title{
Features of the application of one-dimensional equations of hydrodynamics for free-flow flows
}

\author{
Nuriddin Maalem*, and Ilkhom Begmatov \\ Tashkent Institute of Irrigation and Agricultural Mechanization Engineers, Tashkent, Uzbekistan
}

\begin{abstract}
The possibility of applying one-dimensional hydrodynamic equations of a nonstationary flow averaged over the cross-section of the channel during mathematical modeling of long waves-abruptly changing the movement of the water flow in channels is substantiated in the article. The characteristic dimensions of the length are much larger than the depth of the flow-the theory of shallow water. With this averaging, it is necessary to apply some hypotheses, the most important of which is the hypothesis of the distribution of pressure over the depth of the flow
\end{abstract}

\section{Introduction}

In mathematical modeling of the movement of gases and liquids, a system of hydrodynamic equations based on the conservation of mass and momentum is often used[1, 2]. In most cases, they are based on the hypothesis of hydrostatic pressure distribution over depth. It should be noted that in long-wave processes, several important hydrodynamic effects cannot be described by the Saint-Venant equations. Therefore it is necessary, in some way, to take into account the influence on the pressure distribution over the depth of the curvature of the jets [3].

\section{Methods}

For these equations, academician S.A. Khristianovich described internal boundary conditions on Bor, where he used the Galileo transform, a mathematical expression of Newton's 1st law of the jump function [4].

American gas dynamicists R. Courant and K. Friedrichs developed an important idea for various branches of mechanics, according to which the differential equations of motion of the medium are the consequence of integral conservation laws (in the case of gas dynamics - the laws of conservation of mass, momentum, and energy), obtained by applying the Stokes formula to these equations [5]. This formula in a two-dimensional setting is called the green formula.

R. Kurant proved [6] that the integral equations of mechanics are valid for both continuous solutions and discontinuities (in gas dynamics - shock waves). This shows that numerical methods in various sections of mechanics should be based on integral equations, which ensures automatic fulfillment of conditions on discontinuities. Differential equations

*Corresponding author: yuviper29@gmail.com 
obtained in this way are called conservative or divergent.

As is known, the Saint-Venant equations in a channel with an arbitrary form have the form $[7,8,9]$ (here and further, the correction of the amount of motion $\alpha=1$ ):

$$
\begin{gathered}
\frac{\partial \omega}{\partial t}+\frac{\partial Q}{\partial x}=q \\
\frac{\partial Q}{\partial t}+\frac{\partial Q^{2} / \omega+g S}{\partial x}-\left.g \frac{\partial S}{\partial x}\right|_{f_{S}=c}+\frac{\lambda}{2} v^{2} \chi=r, r=\left\{\begin{array}{cc}
q v & q<0 \\
q v_{i n} & q>0
\end{array}\right.
\end{gathered}
$$

where: $\mathrm{t}$ is time, $\mathrm{x}$ is longitudinal (along the bed) coordinate; $\omega$ is the cross-sectional area of water; $Q=\omega v$ is water consumption; v is flow velocity; $S=\omega h_{c}$ is static moment of the cross-section relative to the free surface equals the cross-sectional area to the depth of its centre of gravity vertically $h_{c} ; \chi$ is wetted perimeter; $\mathrm{Z}_{\mathrm{fs}}$ is mark free surface; $\mathrm{g}$ is acceleration of gravity; $q$ is the specific (per unit length of the channel) the flow rate of the inflow when $\mathrm{q}>0$ ("rain intensity") or drawing water from the riverbed when $\mathrm{q}<0 ; v_{\text {in }}$ is the speed of water flowing in from outside (when water flows out of the riverbed in onedimensional schematization, we assume that the momentum carried out of the riverbed flow is related only to the average speed of water in the riverbed), $\lambda=\frac{2 g}{C^{2}}$ is the DarcyWeisbach coefficient of hydraulic friction; C is the Shezi coefficient.

In the practice of computational hydraulics, to set the hydraulic friction vector in twodimensional (planned) equations, the hypothesis is accepted that this vector is collinear to the vector of the depth-averaged flow velocity and is directed in the opposite direction, and to set a specific friction law, standard formulas used for wide rectangular channels are used [10]. Equation (1) is the continuity equation multiplied by the density of the liquid $\mathrm{p}$; it expresses the law of conservation of the mass of the incompressible liquid in the channel. Equation (2) is the equation of motion multiplied by the density of the liquid p; it expresses the law of conservation of momentum. The meaning of some terms of the equation of motion: $Q^{2} / \omega$ is the flow of the amount of movement in the line, divided by the density of water $\rho ; g S$ is hydrostatic pressure in the line (in units of a water column, that is, divided by the density of water $\mathrm{p}) \frac{\partial g S}{\partial x}$ is pressure change along the riverbed; $\frac{\partial g S}{\partial x}-\left.g \frac{\partial S}{\partial x}\right|_{f_{S}}=c$ is part of the pressure change that is not perceived by the riverbed $\left.g \frac{\partial S}{\partial x}\right|_{f_{S}}=c$ is the pressure of the riverbed on the water, $\frac{\lambda}{2} v^{2} \chi$ is hydraulic friction.

In the prismatic channel $\left.g \frac{\partial S}{\partial x}\right|_{f_{s s}=c}$, the term is greatly simplified: $g \frac{\partial S}{\partial x} \mid z_{f_{s}=c}=g \omega I$ and the system takes the form of:

$$
\frac{\partial \omega}{\partial t}+\frac{\partial Q}{\partial x}=q
$$




$$
\frac{\partial Q}{\partial t}+\frac{\partial Q^{2} / \omega+g S}{\partial x}-g \omega I+\frac{\lambda}{2} v^{2} \chi=r, \quad r=\left\{\begin{array}{cc}
q v & q<0 \\
q v_{i n} & q>0
\end{array}\right.
$$

It looks even simpler in the case of a wide rectangular riverbed:

$$
\begin{gathered}
\frac{\partial h}{\partial t}+\frac{\partial \bar{q}}{\partial x}=\hat{q} \\
\frac{\partial \bar{q}}{\partial t}+\frac{\partial \bar{q}^{2} / h+g h^{2} / 2}{\partial x}-g h I+\frac{\lambda}{2} v^{2}=\hat{r}, \quad \hat{r}= \begin{cases}\hat{q} v & \hat{q}<0 \\
\wedge & \wedge \\
q v_{i n} & \hat{q}>0\end{cases}
\end{gathered}
$$

where: $\bar{q}$ is specific flow rate (that is, the flow rate divided by the width of the riverbed In). In this case, the flow rate and the impulse introduced into the channel must also be divided by B: $q=q / B, \hat{r}=r / B$.

Next, consider the equations (1), (2) as:

$$
\begin{gathered}
\frac{\partial \omega}{\partial t}+\frac{\partial v \omega}{\partial x}=q \\
\frac{\partial \omega v}{\partial t}+\frac{\partial v^{2} \omega+g S}{\partial x}-\left.g \frac{\partial S}{\partial x}\right|_{z_{f s}=c}+\frac{\lambda}{2} v^{2} \chi=r
\end{gathered}
$$

We transform equation (8) using the differentiation formula [13]:

$$
\begin{gathered}
(\varphi \psi)^{\prime}=\varphi^{\prime} \psi+\varphi \psi^{\prime} \\
\omega \frac{\partial v}{\partial t}+\left[v \frac{\partial \omega}{\partial t}+v \frac{\partial v \omega}{\partial x}\right]+v \omega \frac{\partial v}{\partial x}+g \frac{\partial S}{\partial h} \frac{\partial h}{\partial x}-g \frac{\partial S}{\partial x} \mid z_{f s}=c \\
+\frac{\lambda}{2} v^{2} \chi=r
\end{gathered}
$$

Moving from the static moment $\mathrm{S}$ to the flow depth $\mathrm{h}$ ( $\mathrm{h}$ is the maximum depth in the line), we assume that $\omega, \mathrm{h}$ and $\mathrm{S}$ are one - to-one functions.

$$
\omega \frac{\partial v}{\partial t}+\left[v \frac{\partial \omega}{\partial t}+v \frac{\partial v \omega}{\partial x}\right]+v \omega \frac{\partial v}{\partial x}+g \frac{\partial S}{\partial h} \frac{\partial h}{\partial x}-g \frac{\partial S}{\partial h} \frac{\partial h}{\partial x} \mid z_{f s}=c+\frac{\lambda}{2} v^{2} \chi=r
$$

From the static moment definition:

$$
\begin{gathered}
\frac{\partial S}{\partial h}=\omega \\
\omega \frac{\partial v}{\partial t}+\left[v \frac{\partial \omega}{\partial t}+v \frac{\partial v \omega}{\partial x}\right]+v \omega \frac{\partial v}{\partial x}+g \omega \frac{\partial h}{\partial x}-g \omega \frac{\partial h}{\partial x} \mid Z_{f s}=c \\
\left.\frac{\partial h}{\partial x}\right|_{Z_{S}}=c \\
=\frac{d Z_{f s}}{d x}=-I
\end{gathered}
$$




$$
\frac{\partial v}{\partial t}+v \frac{\partial v}{\partial x}+g \frac{\partial h}{\partial x}-g I+\frac{\lambda}{2} \frac{v^{2}}{R_{h}}=\frac{r}{\omega}
$$

Equation (14) was widely used in hydraulics until the advantage of using the divergent form of equation (8) became known, and it was in this form that it was given (without the right $\operatorname{part} \frac{r}{\omega}=0$ ).

The following form of the equation of motion can be obtained from equation (14) by obvious identical transformations:

$$
\frac{\partial v}{\partial t}+\frac{\partial v^{2} / 2+g Z f_{s}}{\partial x}+\frac{\lambda}{2} \frac{v^{2}}{R_{h}}=\frac{r}{\omega}
$$

\section{Results and Discussion}

This form of the equation of motion is divergent, but discontinuous (generalized) solutions with borons and hydraulic jumps lead to physically incorrect results. However, it is used in computational hydraulics because burs and hydraulic jumps are not present in the problems under consideration. The advantage of this form of the equation of motion is that with a steady flow, known in hydraulics, the equation of the curve of the free surface in the channel. Numerical methods, as a rule, do not give exact solutions. As a result, we have to resort to some properties that allow us to estimate their approximation to exact solutions. Using the form (15) of the continuity equation, it is possible to achieve (in the absence of bottom roughness and inflow) the exact execution of the Bernoulli equation in the numerical method. This important application of the form (15) was proposed by the famous hydrologist - Professor L. S. Kuchment [11-19].

It should be noted that for the first time, the Saint-Venant equations in divergent form for the simplest special case of a wide rectangular channel were solved at the Institute of Hydrodynamics of the USSR Academy OF Sciences by O. F. Vasiliev and M. T. Gladyshev [12], [20-31].

$$
\left\{\begin{array}{c}
\frac{\partial \omega}{\partial t}+\frac{\partial Q}{\partial x}=q \\
\frac{\partial Q}{\partial t}+\frac{\partial \alpha Q^{2} / \omega+g S}{\partial x}-\left.\frac{\partial g S}{\partial x}\right|_{f_{S}}=\text { const } \\
+\frac{\lambda Q|Q| \chi}{2 \omega^{2}}=0
\end{array}\right.
$$

where: $S=\omega h_{c g}, S$ is the static moment of the live section of the channel relative to the free surface $h_{c g}$ is the depth of immersion of the center of gravity $\chi$ is the wetted perimeter of the channel; $\lambda=\frac{2 g}{C^{2}}$ is the coefficient of hydraulic friction Darcy-Weisbach. The Coriolis coefficient $\alpha$ takes into account the velocity plot. Many scientists were engaged in solving this system of the equation [12-15]. 


\section{Conclusions}

Depending on the problem statement, various restrictions and assumptions were made that simplify the solution to the problem. Therefore, there was a decrease in the correctness of the obtained solutions.

In conclusion, it should be noted that the analysis of all existing solutions of the SaintVenant equation system allowed us to determine the main hypotheses on which the successful solution of one-dimensional Saint-Venant equations is based:

- the depth of the stream must be less than the linear dimensions essential for this engineering task, the flow features along the length of the channel:

$$
h<<L
$$

where: $h$ is the flow depth; $L$ is the characteristic horizontal linear size of the problem to be solved;

- planned (two-dimensional) effects do not affect the flow (but local energy losses due to sharp turns and changes in the shape of the riverbed in the plan can still be taken into account; to account for such losses, increased local hydraulic resistances are introduced in local sections of the riverbed);

- the curvature of the jets in the vertical sections of the flow is small, which allows us to use the hypothesis of hydrostatic pressure distribution over the depth;

- the slope of the free water surface in the direction perpendicular to the flow is small; there is a functional relationship between the mark of the free water surface and the pressure;

- density stratification should not occur in the stream; in river flows, density stratification is rare, but in some cases, its presence occurs (a well-known example is density stratification in a flat river near the bottom water intake of a thermal power plant that discharges partially cooled water into the same river above the water intake);

- when studying nonstationary processes in rivers, it is acceptable to use formulas for the Darcy-Weisbach or Shezi hydraulic friction coefficients derived for the conditions of steady motion in the riverbeds, for example, the Maning or Forchheimer formulas (there are situations when this hypothesis did not correspond to reality);

- corrective amount of movement of $\alpha$ close to 1 (velocity across the target is almost uniform). This hypothesis is not quite correct, even in a wide rectangular channel, when the magnitude of the adjustment affects only the shape of the plot for flow depth $[8,16]$.

It should be noted that the hypotheses justified above and taken as the basis of onedimensional Saint-Venant equations are presented in this interpretation for the first time.

In addition, according to the above, it can be noted that one-dimensional Saint-Venant equations have a phenomenal property - they have infinitely many divergent forms that are equivalent to each other for continuous solutions. Still, each of these forms corresponds to its own hugoniot conditions. Of course, only one form is correct, which is the Galilean transformation of the jump function [17]

\section{References}

1. Zhukovsky N.E. Analogy between the movement of a heavy liquid in a narrow channel and the movement of gas in a pipe at high speed. Collected works, VII

2. Bazarov D., Norkulov B., Vokhidov O., Uljaev F., Ishankulov, Z. Two-dimensional flow movement in the area of protective regulatory structures. IOP Conf. Ser. Mater. Sci. Eng. 890, 012162 (2020)

3. Riabouchinsky D. Sur l'Analogie Hydraulique des Mouvementa d'un Fluide 
Compressible. Compt. Rend. p. 195, (1932)

4. Bazarov D. R., Khidirov S. K., Khaitova D. Possibilities of applying one-dimensional Saint-Venant equations in predictive calculations of sudden failure of a high-pressure dam, 3 (5) pp. 161-166, Almaty, (2014)

5. Khristianovich S.A. unsteady movement in channels and rivers. In: Some new questions of continuum mechanics, pp. 150-154, Moscow, (1938)

6. Stoker J.J. Waves on water, Moscow: publishing house of foreign literature, (1959)

7. Courant R., Friedrichs K. Supersonic current and shock waves. Publishing house of foreign literature, Moscow

8. Bazarov D. and Vokhidov O. Extinguishing Excess Flow Energy in Spillway Structures. In book: Proceedings of EECE 2020, LNCE 150, pp. 535-545, (2021) DOI: 10.1007/978-3-030-72404-7_52

9. Bazarov D. R., Khidirov S. K., Khaitova D. Possibilities of applying one-dimensional Saint-Venant equations in predictive calculations of sudden failure of a high-pressure dam. Bulletin of the Kazakh-German University. Sustainable development of Central Asia 3 (5), pp. 161-166, Almaty, 2014,

10. Bazarov D., Vatin N., Obidov B., and Vokhidov O. Hydrodynamic effects of the flow on the slab of the stand in the presence of cavitation. IOP Conf. Ser. Mater. Sci. Eng. 1030, 012110 (2021)

11. Nureddin M, Begmatov I, Khasanov Kh, and Khidirov S. Dynamics of hydraulic resistance in the zone of constraint of the riverbed, IOP Conf. Ser.: Mater. Sci. Eng. 869 042012, (2020)

12. Kuchment L. S., Demidov V. N., Motovilov Yu. G. Formation of river flow. Physical and mathematical models, Moscow, Nauka, (1983)

13. Vasiliev O.F., Gladyshev M. T. on the calculation of discontinuous waves in open channels. Izv. an SSSR, Mechanics of liquid and gas, № 6, (1966)

14. Fichtenholz G. M. Course of differential and integral calculus, Vol. 2, Moscow, 1969.

15. Bazarov D., Markova I., Sultanov S. and Kattakulov F. Dynamics of the hydraulic and alluvial regime of the lower reaches of the Amudarya after the commissioning of the Takhiatash and Tuyamuyun hydrosystems. IOP Conf. Ser. Mater. Sci. Eng. 1030, 012110 (2021)

16. Maalem N, Khasanov Kh, and Nishanbaev Kh. Morphometric elements of the channel and hydraulic flow parameters in the zone of the river backwater, IOP Conf. Ser.: Mater. Sci. Eng. 8830120136 (2020)

17. Shkolnikov S. Y. Investigation of flows in rivers and lakes by numerical methods, Moscow, (1980)

18. Kochin N.E., Kibel I. A., Roze N.V. Theoretical Hydromechanics, 16 Moscow: Fizmatlit, (1963)

19. Sherenkov I. A. Applied planning problems of open flow hydraulics. Ed. "Energy", M., (1978)

20. Bazarov D., Markova I., Norkulov B., Isabaev K., Sapaeva M. Operational efficiency of water damless intake. IOP Conf. Ser. Mater. Sci. Eng. 869(7), 072051, (2020)

21. Krutov A., Choriev R., Norkulov B., Mavlyanova D. and Shomurodov A. Mathematical modelling of bottom deformations in the kinematic wave approximation. IOP Conf. Ser. Mater. Sci. Eng. 1030, 012147 (2021).

22. Krutov A., Norkulov B., Uljaev F., and Jamalov F. Results of a numerical study of currents in the vicinity of a damless water intake. IOP Conf. Ser. Mater. Sci. Eng. 1030, 012121 (2021).

23. MR Bakiev et al Modelling of horizontal pipe drainage for Sultansandjar dam Journal 
of Physics: Conference Series 1425 (1), (2021)

24. Krutov A., Norkulov B., Mavlyanova D. Simulation of spreading of non-conservative passive substances in water bodies. IOP Conf. Ser. Mater. Sci. Eng. 883(1), 012028 (2020)

25. Krutov A., Norkulov B., Nurmatov P., Mirzaev M. Applicability of zero-dimensional equations to forecast nonconservative components concentration in water bodies. IOP Conf. Ser. Mater. Sci. Eng. 883(1), 012028 (2020)

26. MR Bakiev, EI Kirillova, R Hujaqulov. Safety of hydraulic structures, p. 110. Tashkent, (2008)

27. Krutov A., Norkulov B., Artikbekova F., Nurmatov P. Optimal location of an intake at a reservoir prone to salt diffusion. IOP Conf. Ser. Mater. Sci. Eng. 869(7), 072020, (2020)

28. Khalimbetov, A., Study of submountain river flow patterns constrained by a combined dam. IOP Conference Series: Earth and Environmental Science, 614(1), 012053, (2020)

29. Matyakubov B., Begmatov I., Raimova I. and Teplova G. Factors for the efficient use of water distribution facilities. IOP Conf. Ser. Mater. Sci. Eng. 883, 012025 (2020).

30. Uralov B., Rakhmatov N., Khidirov S., Uljaev F., Raimova I. Hydraulic modes of damless water intake. IOP Conf. Ser. Mater. Sci. Eng. 1030(1), 012123 (2021)

31. Bazarov D., Markova I., Raimova I., Sultanov Sh. Water flow motion in the vehicle of main channels. IOP Conf. Ser. Mater. Sci. Eng. 883, 012025 (2020). 